\title{
A Simple Inducement Scheme to Overcome Adoption Externalities
}

\author{
In-Uck Park \\ Department of Economics, University of Pittsburgh, USA
}

June 2003

\begin{abstract}
Potential customers of network commodities face coordination problems due to adoption externalities that give rise to multiple, Pareto-ranked equilibria. We investigate the extent to which the coordination problem can be resolved by inducement schemes when agents' preferences are private information. Specifically, we show that all symmetric "cut-off strategy" profiles (agents adopt if and only if their type is below a threshold) constitute the set of profiles that can be implemented as a unique equilibrium under an inducement scheme. We derive the ex ante cost of implementing each such profile.

Furthermore, we fully characterize the set of inducement schemes that I) implement each such profile and ii) have the following simple form: each scheme specifies a fixed fee that every adopter pays, and a fixed gross subsidy/prize to be randomly allocated to (or evenly split among) the adopters. We discuss the implications of these findings on the design of optimal schemes for different network organizers, namely, private entrepreneurs and public entities.
\end{abstract}

Keywords: adoption externality, coordination, inducement scheme

JEL Classification: H23, D82

\section{Acknowledgements}

I am very grateful to Jack Ochs for a series of discussions throughout the whole process of developing and writing this paper. I also thank participants at the Midwest Economic Theory Meetings (Spring 2003) for their comments.

\author{
Address for Correspondence \\ Department of Economics \\ University of Pittsburgh \\ WWPH 4P38A \\ Pittsburgh \\ USA \\ ipark@pitt.edu
}




\section{Introduction}

Adoption externalities are inherent in network industries where the utility of consumers from a good/service is positively correlated with the number of other agents who consume the same commodity. In particular, adoption externalities are prominent for commodities that are intrinsically designed for joint consumption or sharing (telephony and data networks), and those that enjoy indirect scale economies for complementary goods (hardware-software and durable-good servicing). They also occur in the diffusion of innovations and standards where compatibility is valuable.

An agent's decision whether to consume/adopt a network commodity critically depends on his belief regarding how many other agents will adopt it. Since positive beliefs lead to positive decisions and vice versa, there tend to be multiple equilibria that are Pareto-ranked: at the worst end is a null equilibrium in which no one adopts because no one is ever anticipated to adopt, while at the other end is a "maximum" equilibrium that refers to a "maximal set of agents" who would indeed adopt when that is what everyone expects. In addition, there may be other intermediate equilibria that are in between these two in terms of both the set of adopters and Pareto criterion. With no outside force present, the particular equilibrium to be realized is indeterminate, a phenomenon known as coordination failure.

As Dybvig and Spatt (1983) clarify, two sorts of public good problems arise in such environments. First, early adopters furnish a public good if the positive externalities they generate launch a domino chain reaction of widespread adoption, possibly leading to the maximum equilibrium. However, agents would be willing to adopt early only if they believe that widespread adoption will follow, an outcome that is not guaranteed. Second, even at the maximum equilibrium the adoption level is below the social optimum because each individual agent's adoption decision does not internalize its positive externality on other adopters.

Dybvig and Spatt (1983) show that these two problems can be resolved by tax/subsidy schemes by a government when the distribution of agents' preferences is known. Specifically, they describe a scheme that effectively works as insurance to adopters against the possibility that fewer agents adopt than in the maximum equilibrium. With this insurance the maximum equilibrium is the unique equilibrium under their scheme (thereby resolving the first problem). This scheme, ingeniously, is costless ex post because the promise of subsidy in unfortunate cases of low adoption ensures that agents adopt without worrying about such cases, thereby preventing the occurrence of such cases. As a consequence, payment of the subsidy is never exercised. Designing such a scheme is possible because the government knows the distribution of agents' preferences and hence the number of adopters in the maximum equilibrium, so that appropriate insurance can be specified precisely when the number of adopters is less than, but not when it is equal to, the maximum equilibrium level. They also provide a separate scheme based on the same logic that guarantees the socially optimal level of adoption as the unique equilibrium (thereby resolving the second problem). However, this scheme is not costless. 
In this paper we consider a related, but different class of environments from that of Dybvig and Spatt, in which the actual distribution of agents' preferences is not known; instead, it is common knowledge that every agent's preference/type is privately drawn from a publicly known distribution. Then, the actual distribution of agents' types is stochastic, hence the maximum equilibrium cannot be known because it depends on the actual distribution of types. Therefore, it is not possible to provide insurance against only the contingencies that fewer agents adopt than in the maximum equilibrium. Moreover, since every agent adopts (does not adopt) when he draws a sufficiently favorable (unfavorable) type, the realized number of adopters can be any number with positive probability and as a result, unlike in Dybvig and Spatt, a subsidy scheme cannot be costless ex post by preventing the occurrence of contingencies that require subsidy payment. As such, the mechanisms of Dybvig and Spatt do not extend to these environments. ${ }^{1}$

We investigate the extent to which the coordination problem can be resolved in this environment by inducement schemes that only affects adopters. Specifically, we show that agents always use "cutoff strategies" (adopt if and only if their type is below a threshold) in equilibrium, and that every symmetric cutoff strategy profile can be implemented as the unique equilibrium under some inducement scheme. For each symmetric cutoff strategy profile, we derive the ex ante cost of implementing it as a unique equilibrium. If the agent's utility is quasi-linear then this cost is invariant to different inducement schemes that may implement it. Furthermore, we fully characterize the set of inducement schemes that i) implement each such profile and ii) have the following simple form: the schemes specify a fixed fee that every adopter pays, and a fixed gross subsidy/prize that is randomly allocated to (or evenly split among) the adopters. The basic intuition behind such schemes is that, since a fixed subsidy is randomly allocated, the fewer adopters there are, the larger is the expected subsidy for each adopter. Hence, this scheme provides a sufficiently high subsidy per adopter to sustain momentum for further adoption, until the threshold type reaches the target level.

These findings carry implications on the optimal schemes that network organizers may desire to employ depending on their objectives. The aforementioned, ex ante cost of implementing an equilibrium is the negative of ex ante profit from the viewpoint of a private entrepreneur. Hence, the optimal scheme for this entrepreneur is one that implements the equilibrium with the lowest ex ante cost. We characterize the common cutoff level of this equilibrium as the solution to a simple maximization problem. This cutoff level is lower than, hence inferior to, in a social welfare sense, that of the maximum equilibrium in the absence of inducement scheme. It may be worth noting that the inducement schemes of the simple form described above exhibit some resemblance to the often-observed private promotion schemes that award prizes to a fixed number of first-arrived subscribers.

\footnotetext{
1 In principle, one can conceive a preference revelation mechanism in which the agents report their realized types to the organizer who then announces the Dybvig-Spatt incentive scheme conditional on the reported type profile. We think it is interesting, for practical as well as theoretical reasons, to examine what can be done when such information collection is not possible.
} 
A public organizer may wish to overcome the coordination failure efficiently (i.e., at minimum cost) by implementing a symmetric equilibrium at least as large as the maximum equilibrium in the absence of inducement scheme. Our results imply that an organizer achieves this goal by implementing this maximum equilibrium in a budget-balanced manner. Note that in spite of the obvious informational disadvantage of the organizer relative to the model of Dybvig and Spatt, we still find that the best equilibrium without inducement can be achieved by a subsidy scheme that is (ex ante) costless. In this sense we strengthen the scope of public intervention suggested by Dybvig and Spatt to remedy the public good problems caused by adoption externalities, although the details of how it may work differ between their environment and ours. We also identify a symmetric social optimum and show that an organizer can implement it, although the scheme that implements it cannot be budget-balanced.

Among existing studies on markets with adoption externalities, a relatively small number investigate inducement schemes to tackle the problem of coordination failure. Before the work of Dybvig and Spatt (1983) detailed above, Rohlfs (1974) considers introductory pricing in a classic paper on telecommunication markets. Bagnoli and Lipman (1989) study a refund mechanism in private contribution to a public project. Andreoni (1998) examines large "leadership gifts" in charitable fund-raising. These papers analyze environments with (almost) complete information, i.e., either the agents' preferences or their distribution are/is known. In a dynamic context of private contribution to a public project, Gradstein (1992) mentions subsidization of early contributors by taxing later contributors as a potential policy instrument to remedy inefficient delay, but he is cautious about the effectiveness of this policy in the presence of incomplete information (of the kind considered in this paper). However, he does not provide an analysis of such schemes.

The remainder of the paper has the following structure. Sections 2 and 3 present and analyze the model without inducement schemes, highlighting the possibility of coordination failure due to adoption externalities. Section 4 presents our main findings on inducement schemes that resolve such problems. Section 5 discusses the implications of the findings on the network organizers depending on their objectives.

\section{Model}

There are $N+1$ ex ante identical agents who i) privately and independently observe their types $t \geq 0$ drawn from a common density function $f: \Re_{+} \rightarrow \Re_{+}$, and then ii) simultaneously choose either to adopt the network product or not. We assume that the cdf $F: \Re_{+} \rightarrow[0,1]$ associated with $f$ is continuous (i.e., $t$ is atomless). For expositional convenience only, we assume $F(t)<1$ for all $t<\infty$ and $f(t)>0$ for all $t>0$.

The payoff to an adopter is determined by his type $t$ and the size of the network formed, measured by the number $n$ of other adopters (i.e., not counting himself): A $t$-type adopter derives a utility of $v_{t}(n) \in \Re$ when there are $n$ other adopters, where $v_{t}(n)$ is a strictly increasing function of $n=0, \cdots, N$. The payoff to a non-adopter is normalized to 0 . Each agent makes an adoption decision to maximize the expected payoff $v_{t}$ of his type. 
An agent's type measures how reluctant he is to join the network, so a higher type means a more conservative agent who needs a larger network to benefit by joining. Hence, it is natural to assume that $v_{t}(n)$ is decreasing in $t$, i.e.,

$$
s<t \Rightarrow v_{s}(n)>v_{t}(n) \quad \forall n=0, \cdots, N .
$$

Specifically, we use the following measurement ${ }^{2}$ : a $t$-type agent for $t \leq N$ is indifferent between adopting and not if all other agents adopt with probability $\frac{t}{N} \leq 1$, i.e., $v_{t}(\cdot)$ satisfies

$$
E\left[v_{t}(n) \mid p\right]:=\sum_{n=0}^{N}\left(\begin{array}{l}
N \\
n
\end{array}\right) p^{n}(1-p)^{N-n} \cdot v_{t}(n) \begin{cases}>0 & \text { if } \frac{t}{N}<p \leq 1 \\
=0 & \text { if } p=\frac{t}{N} \\
<0 & \text { if } 0 \leq p<\frac{t}{N}\end{cases}
$$

Here $E\left[v_{t}(n) \mid p\right]$ is the expected utility of a $t$-type adopter when all others adopt with a probability $p$ : note that this increases strictly in $p$ because the binomial distribution of the number $n$ of adopters for a higher $p$ first-order stochastically dominates that for a lower $p$ and $v_{t}$ is strictly increasing in $n$. Hence, if the equality of (2) for the case $p=\frac{t}{N}$ holds, then so do the inequalities for $\frac{t}{N}<p \leq 1$ and $0 \leq p<\frac{t}{N}$. A natural interpretation of an agent's type, $t$, is the threshold network size above which he is willing to join, in the sense that $t=\frac{t}{N} \cdot N$ is the expected number of other adopters when $p=\frac{t}{N}$. We note that the payoff functions $v_{t}(n)$ that satisfy (1) and (2) are necessarily continuous in $t \in[0, N]$ for each given $n$, as is proved in the Appendix. For expositional convenience, we assume that $v_{t}(n)$ is continuous at all $t \geq 0$ (our basic results are robust to this assumption). A simple example that satisfies (1) and (2) is $v_{t}(n)=n-t$.

An agent $i$ 's strategy is an integrable function (relative to $f$ ) that maps types to adoption probabilities, i.e.,

$$
\sigma^{i}: \Re_{+} \rightarrow[0,1]
$$

where $\sigma^{i}(t)$ is the probability that the agent $i$ adopts (the network product) when his type is $t$. A cutoff strategy (at $\hat{t}$ ) refers to $\sigma^{i}$ such that $\sigma^{i}(t)=1$ if $t<\hat{t}$ and $\sigma^{i}(t)=0$ if $t>\hat{t}$ for some cutoff level $\hat{t} \geq 0$.

\section{Equilibria Without Inducements}

In this section we characterize the (Bayesian Nash) equilibrium of the Bayesian adoption game described above, which is a strategy profile of $N+1$ agents each of whose decision is a best response contingent upon his own type.

Lemma 1: Every agent uses a cutoff strategy in equilibrium.

2 This is without loss of generality (due to rescaling) so long as no type of agent derives positive utility when he is the sole adopter. This is a natural presumption. Moreover, our basic results are robust to this presumption. 
Proof: Each agent $i$ adopts with a probability $p^{i}=\int \sigma^{i}(t) d F$ where $\sigma^{i}$ is the equilibrium strategy of agent $i$. Fix an arbitrary agent, say agent $j$. The distribution $D_{-j}$ on the number $n$ of other agents who adopt is determined by $p^{i}$ s, $i \neq j$. By (1), we deduce that $E_{j}\left[v_{t}(n)\right]$ strictly decreases in $t$ where the expectation is taken relative to $D_{-j}$. Hence, the claim follows since $\sigma^{j}(t)=1$ if $E_{j}\left[v_{t}(n)\right]>0$ and $\sigma^{j}(t)=0$ if $E_{j}\left[v_{t}(n)\right]<0$.

Lemma 2: All agents use the same cutoff strategy in equilibrium.

Proof: By Lemma 1 let $\hat{t}^{i} \geq 0$ be the cutoff level of agent $i$, so he adopts with probability $p^{i}=F\left(\hat{t}^{i}\right)$. Suppose, contrary to the claim, that two agents $j$ and $k$ have different cutoff levels, say $\hat{t}^{j}<\hat{t}^{k}$. Let $D_{-i}$ denote the distribution on the number $n$ of adopters except the agent $i: D_{-i}$ is determined by the probabilities that other agents adopt. Note that $D_{-j}$ first-order stochastically dominates $D_{-k}$ because $p^{j}<p^{k}$. It follows, therefore, that $E_{j}\left[v_{t}(n)\right]>E_{k}\left[v_{t}(n)\right]$ for every $t$, hence for $\hat{t}^{j}<t<\hat{t}^{k}$ in particular, where $E_{i}\left[v_{t}(n)\right]$ is the expected value of $v_{t}(n)$ relative to $D_{-i}$. But, this is impossible because $\hat{t}^{j}<\hat{t}^{k}$ implies that $E_{j}\left[v_{t}(n)\right]<0$ and $E_{k}\left[v_{t}(n)\right]>0$ for $\hat{t}^{j}<t<\hat{t}^{k}$.

By virtue of Lemma 2 we represent an equilibrium by a common cutoff level $\hat{t}$ : an agent prefers to adopt precisely when his type is below $\hat{t}$, given that all other agents adopt precisely when their types are below $\hat{t}$. Since $v_{t}(n)$ is continuous in $t$, this is the case if a $\hat{t}$-type agent is indifferent between adopting and not. That is, a cutoff level $\hat{t}$ is an equilibrium one if and only if

$$
V(\hat{t}):=E\left[v_{\hat{t}}(n) \mid p=F(\hat{t})\right]=0 .
$$

Since $V(0)=0$ by (2), $\hat{t}=0$ is an equilibrium cutoff level which depicts the "nocoordination" equilibrium in which no agent adopts precisely because the belief that no one would ever adopt is self-fulfilling. On the other hand, for all $\hat{t} \geq N$ we have $V(\hat{t})<$ $E\left[v_{\hat{t}}(n) \mid p=1\right]<E\left[v_{N}(n) \mid p=1\right]=0$ where the first and second inequalities follow from $F(\hat{t})<1$ and (1), respectively, and the final equality follows from (2). This means that no $\hat{t} \geq N$ can be an equilibrium cutoff level because such types would not adopt even if all other agents adopt with certainty. Since $v_{t}$ is continuous so is $V(t)$ and, therefore, there is a maximum type, denoted by $t^{*}<N$, such that $V\left(t^{*}\right)=0$, hence $V(\hat{t})<0$ for all $\hat{t}>t^{*}$. We refer to $t^{*}$ as the maximum equilibrium (cutoff level) without inducement scheme.

We say that a cutoff level $t$ is "under expansion (contraction) pressure" if $V(t)>0$ $(V(t)<0)$ in the sense that if all agents are believed to use the same cutoff strategy at level $t$, all of them would find it beneficial to increase (decrease) their own cutoff levels. A typical coordination problem with adoption externalities is that a pessimistic anticipation of adoption rate (i.e., short of the "critical mass") is reinforced by contraction pressure toward an even lower adoption rate, although once the anticipation is pushed beyond the critical mass for some reason the expansion pressure would be self-reinforcing until the network grows to the maximum size. Since we are interested in identifying possible schemes that would overcome such coordination problems, below we consider environments that are 
subject to such problems. Our analysis and findings apply more generally, however it may be expositional useful to focus on situations in which there is a type $t^{m}$ strictly between 0 and $t^{*}$ such that

$$
\text { i) } \left.V\left(t^{m}\right)=0 \text {, ii) } V(t)<0 \text { for all } t \in\left(0, t^{m}\right) \text {, and } i i i\right) V(t)>0 \text { for all } t \in\left(t^{m}, t^{*}\right) \text {. }
$$

Here, $t^{m}$ represents the critical mass in the sense that if agents believe that all other agents use cutoff strategies at levels $t^{m}$ or above (some strictly above), then the expansion pressure continues to increase the agents' cutoff levels all the way up to $t^{*}$, i.e., to the maximum equilibrium. On the other hand, there are two other equilibria with lesser degrees of coordination, one in which the equilibrium cutoff level is $t^{m}$ and the other in which no one ever adopts.

As a simple example, note that (4) is satisfied for all large $N$ when $v_{t}(n)=n-t$ and $f$ is single-peaked with $f(0)=0$. In this case $V(t)$ is strictly convex for lower values of $t$ and is strictly concave for higher value of $t$, with three equilibrium cutoff levels, namely, $0, t^{m}$ and $t^{*}$, as illustrated in Figure 1 .

[Figure 1 about here]

\section{Inducement Schemes}

An inducement scheme is a rule that assigns a lottery (a probability distribution of monetary transfers) to each agent contingent on the observable outcome of the adoption game, i.e., on the set of adopters. Since all agents are identical for an organizer at the point in time that the organizer sets an inducement scheme, it is natural to consider inducements schemes that are "anonymous" in the sense that the lottery assignment rules do not distinguish agents with identical observable characteristics. ${ }^{3}$ Following Dybvig and Spatt (1983), we postulate that an agent of type $t$ tries to maximize the expected value of a quasi-linear utility function $v_{t}(n)+s$ where $v_{t}(n)$ is the utility from adopting the network as described earlier and $s$ is the net transfer/subsidy to him according to the inducement scheme in place.

The quasi-linear specification implies that the agents are only concerned about the expected value of the lotteries (i.e., not about the precise distributions of the prizes) assigned by an inducement scheme. The organizer is concerned about the ex ante running cost of a scheme, hence what matters to the organizer is also the expected values of the lotteries. Therefore, we may represent a general (anonymous inducement) scheme by a function $s_{A}:\{0, \cdots, N+1\} \rightarrow \Re$ where $s_{A}(\kappa)$ is the net expected monetary transfer to each adopter when the number of all adopters is $\kappa \in\{0, \cdots, N+1\}$. (Note that we do not consider monetary transfers to/from non-adopters, because any such transfer that may facilitate adoption would be taxing the non-adopters which is infeasible for organizers

3 Dybvig and Spatt (1983) also consider anonymous inducement schemes. 
without taxing authority; even if feasible, it would be unpopular as a public policy, as well as unrealistic.)

An equilibrium under a scheme $s_{A}$ is an equilibrium of the Bayesian adoption game when all the agents know that the scheme $s_{A}$ is in place. It is straightforward to verify that Lemma 1 still holds for equilibria under a scheme $s_{A}$, hence every agent uses a cutoff strategy.

If an inducement scheme is in place Lemma 2 may not hold generally and equilibria may exist in which agents use different cutoff strategies: an agent could have a higher cutoff level than another because the lower expected utility (from network good) of the former is exactly cancelled off by a higher expected net subsidy than the latter (both disparities due to the former facing a "lower" distribution of the number of other adopters). We believe that such equilibria are less focal than symmetric ones because they would require identical agents to have different beliefs that are delicately related to satisfy equilibrium conditions. Achieving such asymmetric coordination would be an extreme coincidence especially because the identities of the agents holding different beliefs within equilibrium are completely arbitrary, hence there are many asymmetric equilibria that differ only in artificial naming of the agents. For these reasons, asymmetric equilibria would be particularly uncertain and unattractive goals to target for an organizer using inducement schemes. Furthermore, the above observation establishes that if there is a unique equilibrium under a scheme it must be a symmetric one.

In the sequel, therefore, we will examine inducement schemes under which there is a unique symmetric equilibrium, say with a common cutoff level $\hat{t}$. By the same reasoning as that leading to the equilibrium characterization (3), $\hat{t}$ is a symmetric equilibrium cutoff level under $s_{A}$ if and only if

$$
V(\hat{t})+E\left[s_{A}(\kappa) \mid p=F(\hat{t})\right]=0
$$

where $E\left[s_{A}(\kappa) \mid p=F(\hat{t})\right]$ is the expected value of $s_{A}(\kappa)$ for an agent when he adopts given that all other agents adopt with probability $F(\hat{t})$.

Lemma 3: If $\hat{t}$ is the common cutoff level of a symmetric equilibrium under a scheme $s_{A}$, the ex ante cost of inducing this equilibrium is

$$
C(\hat{t}):=\sum_{\kappa=0}^{N+1}\left(\begin{array}{c}
N+1 \\
\kappa
\end{array}\right) F(\hat{t})^{\kappa}(1-F(\hat{t}))^{N+1-\kappa} s_{A}(\kappa) \kappa=-(N+1) F(\hat{t}) V(\hat{t})
$$

Proof: By definition,

$$
E\left[s_{A}(\kappa) \mid p=F(\hat{t})\right]:=\sum_{n=0}^{N}\left(\begin{array}{l}
N \\
n
\end{array}\right) F(\hat{t})^{n}(1-F(\hat{t}))^{N-n} s_{A}(n+1)
$$


Hence,

$$
\begin{aligned}
C(\hat{t}) & =\sum_{\kappa=1}^{N+1} \frac{(N+1) !}{\kappa !(N+1-\kappa) !} F(\hat{t})^{\kappa}(1-F(\hat{t}))^{N+1-\kappa} s_{A}(\kappa) \kappa \\
& =(N+1) F(\hat{t})\left[\sum_{\kappa=1}^{N+1} \frac{N !}{(\kappa-1) !(N+1-\kappa) !} F(\hat{t})^{\kappa-1}(1-F(\hat{t}))^{N+1-\kappa} s_{A}(\kappa)\right] \\
& =(N+1) F(\hat{t})\left[\sum_{n=0}^{N} \frac{N !}{n !(N-n) !} F(\hat{t})^{n}(1-F(\hat{t}))^{N-n} s_{A}(n+1)\right] \\
& =(N+1) F(\hat{t}) E\left[s_{A}(\kappa) \mid p=F(\hat{t})\right] \\
& =-(N+1) F(\hat{t}) V(\hat{t}) .
\end{aligned}
$$

Here, the second equality is obtained by factoring out $(N+1) F(\hat{t})$, the third one by change of variables (i.e., $n=\kappa-1$ ), and the last two follow from (7) and (5), respectively.

This lemma specifies the expected total net transfer to the agents, $C(\hat{t})$, in case there is a scheme $s_{A}$ under which the symmetric cutoff strategies at $\hat{t}$ constitute an equilibrium. Note that it is independent of $s_{A}$ : a net transfer of $-V(\hat{t})$ for each adopter is required for a $\hat{t}$-type agent to be the marginal type to adopt, and each agent incurs this cost with a probability $F(\hat{t})$, hence the right hand side of $(6)$.

We now show that for any $\hat{t}>0$ there indeed exists a scheme $s_{A}$ under which the symmetric cutoff strategies at $\hat{t}$ constitute a unique symmetric equilibrium: in this case we say that $s_{A}$ implements a symmetric equilibrium at cutoff level $\hat{t}$ (or, implements a common cutoff level $\hat{t}$, for short). In particular, we identify such schemes that have the following simple form: The organizer sets a fee $T \geq 0$ that every adopter pays and a total subsidy/prize $S \geq 0$ that is randomly allotted to one adopter (splitting $S$ evenly among all adopters has the same effect due to quasi-linear specification). This scheme is represented by $s_{A}(\kappa)=(S / \kappa)-T$ for $\kappa>0$ and $s_{A}(0)=0$. For notational simplicity, however, we use $(S, T)$ as shorthand for such a scheme.

Given a scheme $(S, T)$, let

$$
P_{A}(t):=\sum_{n=0}^{N}\left(\begin{array}{l}
N \\
n
\end{array}\right) F(t)^{n}(1-F(t))^{N-n} \frac{1}{n+1}
$$

denote the probability that an adopter "wins" (i.e., gets allotted) the prize $S$ given that all other agents adopt precisely when their type is below $t$, so that the expected subsidy amount is $P_{A}(t) \cdot S$ and, therefore, the expected overall surplus of a $t$-type adopter under $(S, T)$ is

$$
W(t \mid S, T):=V(t)+P_{A}(t) \cdot S-T .
$$

Note that $W(\hat{t} \mid S, T)=0$ is the condition (5) for $\hat{t}$ to be a symmetric equilibrium cutoff level under $(S, T)$. If $(S, T)$ implements a common cutoff level $\hat{t}$, therefore, $W(\hat{t} \mid S, T)=0$ and $W(t \mid S, T) \neq 0$ for all $t \neq \hat{t}$. 
Given $\hat{t}>0$, for any $S>0$ one can choose $T$ to ensure $W(\hat{t} \mid S, T)=0$, i.e., $T=$ $V(\hat{t})+P_{A}(\hat{t}) \cdot S$. Since $P_{A}(t)$ is decreasing in $t$, the higher $S$ is the more rapidly decreasing is the part $P_{A}(t) \cdot S-T$ of (10) in $t$. Note that $V(t)$ is bounded on the closed interval $[0, \hat{t}]$. With $T$ varying to maintain $W(\hat{t} \mid S, T)=0$, therefore, one can find a lower bound, say $\underline{S}^{\prime}$, such that if $S>\underline{S}^{\prime}$ then $P_{A}(t) \cdot S-T$ exceeds $-V(t)$, i.e., $W(t \mid S, T)>0$, for all $0 \leq t \leq \hat{t}{ }^{4}$ Furthermore, since $V(t)$ is bounded above for $t>\hat{t}$, there is a lower bound, say $\underline{S}^{\prime \prime}$, such that if $S>\underline{S}^{\prime \prime}$ then $-P_{A}(t) \cdot S+T$ exceeds $V(t)$, i.e., $W(t \mid S, T)<0$, for all $t>\hat{t}$. Hence, $\hat{t}$ is a unique symmetric equilibrium cutoff level under $(S, T)$ if $S>\max \left\{\underline{S}^{\prime}, \underline{S}^{\prime \prime}\right\}$. This result is formalized in the next theorem.

Theorem 1: For any $\hat{t}>0$ there is a lower bound $\underline{S}(\hat{t}) \geq 0$ such that the scheme $(S, T)$ implements the symmetric equilibrium at cutoff level $\hat{t}$ if and only if $S>\underline{S}(\hat{t})$ and $T=V(\hat{t})+P_{A}(\hat{t}) \cdot S$.

A proof is provided in the Appendix. Instead, we give a graphical illustration of the level of $\underline{S}(\hat{t})$ when $\hat{t}=t^{*}$ in Figure 1. Given $S>0$, the graph of $-P_{A}(t) \cdot S<0$ is increasing in $t$. As $S$ increases, the vertical distance from the horizontal axis to the graph of $-P_{A}(t) \cdot S<0$ increases proportionately, i.e., the graph moves downward and the slope gets steeper. $T=V\left(t^{*}\right)+P_{A}\left(t^{*}\right) \cdot S$ is represented by the vertical distance from $V(t)$ to $-P_{A}(t) \cdot S$ at $t=t^{*}$. The level of $\underline{S}\left(t^{*}\right)$ is one such that this distance from $V(t)$ to $-P_{A}(t) \cdot \underline{S}\left(t^{*}\right)$ at $t=t^{*}$ coincides with the shortest distance from $V(t)$ to $-P_{A}(t) \cdot \underline{S}\left(t^{*}\right)$ for $0 \leq t \leq t^{m}$, as illustrated in Figure 1. By shifting the graph of $-P_{A}(t) \cdot S$ upward by $T$ so that it goes through $t^{*}$, we get the graph of $T-P_{A}(t) \cdot S$. For $S>\underline{S}\left(t^{*}\right)$, note that this graph lies below $V(t)$ for $0 \leq t<t^{*}$, so that $W(t \mid S, T)>0$. In the current case of $\hat{t}=t^{*}$, for all $t>t^{*}$ this graph clearly lies above $V(t)$ because $V(t)<V\left(t^{*}\right)=0$, hence $W(t \mid S, T)<0$. For other cases where $V(t)>V(\hat{t})$ for some $t>\hat{t}$, one also needs to choose $S$ sufficiently large to ensure that the graph of $T-P_{A}(t) \cdot S$ lies above $V(t)$ for all $t>\hat{t}$.

Our discussion so far is based on an implicit presumption that if there are multiple equilibria and only one of them is symmetric, the latter is the focal one that will be realized. Although we believe that this is a plausible presumption as argued earlier, we emphasize that our result is robust to this presumption. In particular, for sufficiently large $S$, the scheme $(S, T)$ in Theorem 1 implements the common cutoff level $\hat{t}$ as the unique equilibrium, symmetric or not, under minor technical assumptions, as the next result states.

Theorem 2: Suppose $v_{t}(n)$ is differentiable in $t$ for each $n$, and is bounded, i.e., $\left|v_{t}(n)\right|<M$ for all $t$ and $n$ where $M<\infty$. For any $\hat{t}>0$ there is a threshold $S^{*}(\hat{t}) \geq \underline{S}(\hat{t})$ such that, if $S>S^{*}(\hat{t})$ and $T=V(\hat{t})+P_{A}(\hat{t}) \cdot S$, the unique equilibrium under the scheme $(S, T)$ is a symmetric one in which all agents use cutoff strategies at $\hat{t}$.

Proof: See Appendix.

4 For this conclusion it is also needed that $-V(t)$ is not decreasing at an "infinite rate" at $\hat{t}$, which is proved in the Appendix. 


\section{Discussion}

In the previous section we showed that the ex ante cost of inducing a symmetric equilibrium with cutoff level $\hat{t}$, if possible under an inducement scheme, is $C(\hat{t})$ in $(6)$; and we established existence of such schemes for every $\hat{t}>0$, by identifying a simple class of schemes that implement this equilibrium. These findings carry some implications on the optimal schemes that a network organizer may desire to employ depending on the objectives.

\subsection{Private Entrepreneur}

For a business entrepreneur operating a network industry for profit, the negative of $C(\hat{t})$ in Lemma $3,(N+1) F(\hat{t}) V(\hat{t})$, is the expected profit when she implements the symmetric equilibrium with cutoff level $\hat{t}$ by a scheme. Hence, the maximum possible profit is $(N+1) F\left(t^{\pi}\right) V\left(t^{\pi}\right)$ where $t^{\pi} \in \operatorname{argmax}_{t \geq 0} F(t) V(t)$. This maximum profit can be achieved by a simple scheme $(S, T)$ with $S>\underline{S}\left(t^{\pi}\right)$ and $T=V\left(t^{\pi}\right)+P_{A}\left(t^{\pi}\right) \cdot S$. This scheme has a natural interpretation: $T$ is a uniform price charged to every customer and $S$ is a promotion prize that is allocated to one customer by lottery. Clearly, $V\left(t^{\pi}\right)>0$. Hence, $t^{\pi} \in\left(t^{m}, t^{*}\right)$ in environments that satisfy (4). Summarizing, we have

Corollary 1: A network organizer maximizes ex ante profit by implementing a common cutoff level $t^{\pi}$ that solves $\max _{t \geq 0} F(t) V(t) . t^{\pi} \in\left(t^{m}, t^{*}\right)$ and the maximum ex ante profit is $(N+1) F\left(t^{\pi}\right) V\left(t^{\pi}\right)>0$.

Note that there is a continuum of schemes $(S, T)$ that achieve the maximum ex ante profit. ${ }^{5}$ This indeterminacy can be alleviated by some additional criteria that the entrepreneur may desire to meet. For instance, for credibility reasons the entrepreneur may want to ensure that potential ex post loss is within a certain limit, say $\ell$. Since the scheme would incur largest loss when only one agent adopts, if $V\left(t^{\pi}\right)+\left(P_{A}\left(t^{\pi}\right)-1\right) \cdot \underline{S}\left(t^{\pi}\right)>-\ell$, then the entrepreneur can ensure this limit with $(S, T)$ by selecting $S$ sufficiently close to $\underline{S}\left(t^{\pi}\right)$. Otherwise, she cannot ensure this limit by a scheme of the simple form considered in Theorem 1. Instead, she may look for a more complex scheme that reduces the loss in case of low adoption (still satisfying (5) only when $\hat{t}=t^{\pi}$ ), e.g., by offering an appropriate number of smaller prizes to be randomly allocated to adopters. This scheme more closely resembles the often-observed promotion schemes that award prizes to a fixed number of first-arrived subscribers.

\subsection{Public Organizer}

Sometimes public entities may promote network commodities, e.g., in such cases as diffusion of innovations and standards. The internet was also sponsored initially by the

5 Technically, this multiplicity arises primarily because the agents are risk-neutral in monetary transfers due to the quasi-linear specification. If this risk-neutrality is relaxed, efficient inducement schemes will be more complex but still be based on the same basic principle of providing sufficient subsidy per adopter when there are fewer adopters. In case the agents are risk-averse, an efficient scheme $s_{A}(\kappa)$ will be much less sensitive to $\kappa$ than $(S, T)$ is, to save in subsidy payments by reducing the agents' risk. 
government. We consider two possible objectives that public entities may pursue as a network organizer.

First, consider an organizer that is interested in the most efficient (i.e., minimal cost) ways to overcome the coordination failure by implementing a symmetric equilibrium with cutoff level at least $t^{*}$ (i.e., at least as big as the maximum equilibrium in the absence of inducement scheme). Since $V(t)<0$ for $t>t^{*}$, Lemma 3 implies that the lower bound on the ex ante cost of implementing a common cutoff level $t^{*}$ is 0 , i.e., in a budget-balanced manner; and implementing any larger common cutoff level is more costly. Theorems 1 and 2 establish that the organizer can indeed implement $t^{*}$ as the common cutoff level (the maximum equilibrium in the absence of inducement scheme) at a balanced budget.

Alternatively, an organizer may wish to implement the social optimum and want to know the cost. To identify the social optimum, observe that an agent increases total social welfare by adopting if the externality he exerts on others exceeds the utility loss to himself. Since the utility loss increases with his type while the generated externality is independent of it, all agents use a cutoff strategy at social optimum. Given a symmetric strategy profile with a common cutoff level $\hat{t}$, the ex ante expected utility of an agent is $E V(\hat{t}):=$ $\int_{t \leq \hat{t}} V(t \mid \hat{t}) d F$ where $V(t \mid \hat{t})=\sum_{n=0}^{N}\left(\begin{array}{l}N \\ n\end{array}\right) F(\hat{t})^{n}(1-F(\hat{t}))^{N-n} v_{t}(n)$ is the expected utility of a $t$-type adopter. A social optimum is a common cutoff level $t^{\mathrm{so}}$ such that $E V\left(t^{\mathrm{so}}\right) \geq E V(\hat{t})$ for all $\hat{t}^{6}$ It is clear that $E V(\hat{t})<E V\left(t^{*}\right)$ if $\hat{t}<t^{*}$ because $V(t \mid \hat{t})<V\left(t \mid t^{*}\right)$ for all $t<\hat{t}$ and $V\left(t \mid t^{*}\right)>0$ for $\hat{t}<t<t^{*}$. It is also clear that $t^{\text {so }} \neq t^{*}$ because a $t^{*}$-agent is indifferent between adopting and not when all others cutoff at $t^{*}$, hence types in a neighborhood of $t^{*}$ should adopt when the externality is considered. Therefore, $t^{\text {so }}>t^{*}$. We know from the previous section that this social optimum can be implemented at an ex ante deficit of $C\left(t^{\mathrm{so}}\right)=-(N+1) F\left(t^{\mathrm{so}}\right) V\left(t^{\mathrm{so}}\right)$. These findings (summarized in the next corollary) extend the scope of inducement schemes as a public means to overcome adoption externalities, suggested by Dybvig and Spatt (1983), to situations where the organizer has incomplete information on the distribution of customers' preferences.

Corollary 2: A network organizer can implement the common cutoff level $t^{*}$ in an ex ante budget-balanced manner: this is the least costly way of inducing a symmetric equilibrium with cutoff level $t^{*}$ or higher. An organizer can implement the social optimum cutoff level, $t^{\mathrm{so}}$, at an ex ante deficit of $-(N+1) F\left(t^{\mathrm{so}}\right) V\left(t^{\mathrm{so}}\right)>0$.

Finally, although we carried out the analysis in a static setting, the issue is intrinsically dynamic and, therefore, extending the investigation to dynamic settings will be fruitful in understanding the network-formation mechanism more fully. We are currently investigating the effect on final network size of having the number of agents already adopted available

6 In view of our argument earlier on the impracticability of implementing asymmetric strategy profiles as equilibrium, we define social optimum among all symmetric strategy profiles. It is not obvious, although tempting to conjecture, that a social optimum strategy profile is necessarily symmetric, because spreads in cutoff levels can reduce the variance of final network sizes by rendering extreme realizations less likely, which could be beneficial for some utility functions. One can show that this conjecture is true is when the externality exhibits increasing returns to scale, i.e., $v_{t}(n+1)-v_{t}(n) \geq v_{t}(n)-v_{t}(n-1)$. 
as public information, when the agents have multiple chances to adopt. ${ }^{7}$

\section{Appendix}

Proof of continuity of $v_{t}(n)$ : We prove our earlier assertion (in Section 2) that the payoff functions $v_{t}(n)$ that satisfy (1) and (2) are continuous in $t \in[0, N]$ for each given $n=$ $0, \cdots, N$. First, to show left-continuity, consider $0<s \leq N$. By definition, $E\left[v_{s-\epsilon}(n) \mid p=\right.$ $(s-\epsilon) / N]=0$ for all $\epsilon$ in a small interval $[0, \bar{\epsilon}]$. If $v_{s}(n) \neq \lim _{t \uparrow s} v_{t}(n)$ for some $n$, then $v_{s}(n)<\lim _{t \uparrow s} v_{t}(n)$ because $v_{t}(n)$ is decreasing in $t$. Since the binomial probabilities $\left(\begin{array}{l}N \\ n\end{array}\right) p^{n}(1-p)^{N-n}$ is continuous in $p$ for each $n$, such discontinuity of $v_{t}(n)$ would mean that $E\left[v_{s-\epsilon}(n) \mid p=(s-\epsilon) / N\right]$, as a function of $\epsilon$, is discontinuous at $\epsilon=0$, contradicting our earlier assertion that it is identically 0 for $\epsilon \in[0, \bar{\epsilon}]$. This establishes that $v_{t}(n)$ must be left-continuous at all $0<t \leq N$ for each $n$. An analogous argument establishes that $v_{t}(n)$ must be right-continuous at all $0 \leq t<N$ for each $n$, thereby proving the claim.

Proof of Theorem 1: Fix $\hat{t}>0$ and let $L(\hat{t}):=\{0 \leq t<\hat{t} \mid V(t) \leq V(\hat{t})\}$. For each $\tau \in$ $L(\hat{t})$ let $\underline{S}^{\prime}(\tau)$ denote the unique value of $S$ that solves $\left(P_{A}(\tau)-P_{A}(\hat{t})\right) \cdot S+V(\tau)-V(\hat{t})=0$ : Since $P_{A}(\cdot)$ is a decreasing function, $\underline{S}^{\prime}(\tau) \geq 0$ and $\left(P_{A}(\tau)-P_{A}(\hat{t})\right) \cdot S+V(\tau)-V(\hat{t})>(<) 0$ if $S>(<) \underline{S}^{\prime}(\tau)$. Define $\underline{S}^{\prime}:=\sup _{\tau \in L(\hat{t})} \underline{S}^{\prime}(\tau)$. Let $\underline{S}^{\prime}=0$ if $L(\hat{t})=\emptyset$.

We now show that $\underline{S}^{\prime}$ is finite. This is trivial if $\hat{t}$ is not a limit point of $L(\hat{t})$, because then $P_{A}(\tau)-P_{A}(\hat{t})>0$ is bounded away from 0 and $V(\tau)-V(\hat{t})$ is bounded for all $\tau \in L(\hat{t})$. Suppose $\hat{t}$ is a limit point of $L(\hat{t})$. Note that $f(\hat{t})>0$ for $\hat{t}>0$ by assumption. ${ }^{8}$ Hence, $P_{A}(t)$ has a finite, non-zero derivative at $t=\hat{t}$ (Cf. (9)). Furthermore, since $v_{t}(n)$ decreases in $t$ and $\left(\begin{array}{l}N \\ n\end{array}\right) F(t)^{n}(1-F(t))^{N-n}$ has a finite derivative at $t=\hat{t}$ for each $n$, the rate at which $V(t)=\sum_{n=0}^{N}\left(\begin{array}{c}N \\ n\end{array}\right) F(t)^{n}(1-F(t))^{N-n} v_{t}(n)$ increases is bounded, i.e., $\frac{V(\hat{t})-V(t)}{\hat{t}-t}<K$ for some $K>0$ in a small neighborhood of $\hat{t}$. Hence, $\underline{S}^{\prime}(\tau)=\frac{V(\hat{t})-V(\tau)}{P_{A}(\tau)-P_{A}(\hat{t})}$ is bounded above for all $\tau \in L(\tau)$ in a small neighborhood of $\hat{t}$ and, therefore, $\underline{S}^{\prime}$ is finite.

By construction of $\underline{S}^{\prime}$, we have

i) If $S>\underline{S}^{\prime}$, then $\left(P_{A}(t)-P_{A}(\hat{t})\right) \cdot S+V(t)-V(\hat{t})>0$ for all $t \in L(\hat{t})$;

ii) If $S=\underline{S}^{\prime}$, then $\left(P_{A}(t)-P_{A}(\hat{t})\right) \cdot S+V(t)-V(\hat{t})>0$ for all $t \in L(\hat{t})$ if and only if $\underline{S}^{\prime}=\lim _{t \uparrow \hat{t}} \underline{S}^{\prime}(t)>\underline{S}^{\prime}\left(t^{\prime}\right)$ for all $t^{\prime}<\hat{t}$;

7 Existing studies of dynamic coordination problems include Rohlfs (1974) on telecommunication markets, Farrell and Saloner (1985) on diffusion of new standards, Gale (1995) on investments with strategic complementarities, and Bagnoli and Lipman (1989), Gradstein (1992) and Marx and Matthews (2000) on private provision of funds to a public project.

$8 \underline{S}^{\prime}$ can be proved finite even if $f(\hat{t})=0$ so long as there does not exist a sequence $\left\{t_{k}\right\}$ converging to $\hat{t}$ such that $\lim _{k \rightarrow \infty} \frac{v_{\hat{t}}(n)-v_{t_{k}}(n)}{\hat{t}-t_{n}}=0$ for all $n=0, \cdots, N$, because then $\hat{t}$ cannot be a limit point of $L(\hat{t})$. It is straightforward to prove that this condition is satisfied at all $\hat{t} \in[0, N]$ for $v_{t}(n)$ that satisfy (1) and (2); For $t>N$, being a strictly decreasing function, $v_{t}(n)$ fails to have a strictly negative derivative, hence fails to satisfy this condition, only in a set of measure zero. Without the assumption $f(\hat{t})>0$, therefore, $\underline{S}^{\prime}$ can still be proved to be finite, hence Theorem 1 , for all $0<\hat{t} \leq N$ and for generic $\hat{t}>N$. 
iii) If $0<S<\underline{S}^{\prime}$, then $\left(P_{A}(t)-P_{A}(\hat{t})\right) \cdot S+V(t)-V(\hat{t})<0$ for some $t \in L(\hat{t})$;

iv) $\left(P_{A}(t)-P_{A}(\hat{t})\right) \cdot S+V(t)-V(\hat{t})>0$ for all $t<\hat{t}$ such that $t \notin L(\hat{t})$.

Analogously, let $U(\hat{t}):=\{t>\hat{t} \mid V(t) \geq V(\hat{t})\}$. For each $\tau \in U(\hat{t})$ let $\underline{S}^{\prime \prime}(\tau)$ be the unique value of $S$ that solves $\left(P_{A}(\tau)-P_{A}(\hat{t})\right) \cdot S+V(\tau)-V(\hat{t})=0$ : Since $P_{A}(\cdot)$ is a decreasing function, $\underline{S}^{\prime \prime}(\tau) \geq 0$ and $\left(P_{A}(\tau)-P_{A}(\hat{t})\right) \cdot S+V(\tau)-V(\hat{t})<(>) 0$ if $S>(<) \underline{S}^{\prime \prime}(\tau)$. Define $\underline{S}^{\prime \prime}:=\sup _{\tau \in U(\hat{t})} \underline{S}^{\prime \prime}(\tau)$. Let $\underline{S}^{\prime \prime}=0$ if $U(\hat{t})=\emptyset$. It can be shown that $\underline{S}^{\prime \prime}$ is finite in a manner analogous to before. Again, by construction,

v) If $S>\underline{S}^{\prime \prime}$, then $\left(P_{A}(t)-P_{A}(\hat{t})\right) \cdot S+V(t)-V(\hat{t})<0$ for all $t \in U(\hat{t})$;

vi) If $S=\underline{S}^{\prime \prime}$, then $\left(P_{A}(t)-P_{A}(\hat{t})\right) \cdot S+V(t)-V(\hat{t})<0$ for all $t \in U(\hat{t})$ if and only if $\underline{S}^{\prime \prime}=\lim _{t \downarrow \hat{t}} \underline{S}^{\prime \prime}(t)>\underline{S}^{\prime \prime}\left(t^{\prime}\right)$ for all $t^{\prime}>\hat{t}$

vii) If $S<\underline{S}^{\prime \prime}$, then $\left(P_{A}(t)-P_{A}(\hat{t})\right) \cdot S+V(t)-V(\hat{t})>0$ for some $t \in U(\hat{t})$;

viii) $\left(P_{A}(t)-P_{A}(\hat{t})\right) \cdot S+V(t)-V(\hat{t})<0$ for all $t>\hat{t}$ such that $t \notin U(\hat{t})$.

Define $\underline{S}(\hat{t}):=\max \left\{\underline{S}^{\prime}, \underline{S}^{\prime \prime}\right\}$. It follows from i), iv), v) and viii) that if $S>\underline{S}(\hat{t})$ then $W(t \mid S, T)>0$ for all $t<\hat{t}, W(\hat{t} \mid S, T)=0$, and $W(t \mid S, T)<0$ for all $t>\hat{t}$, establishing the "if" part of the claim.

To prove the converse, consider $(S, T)$ with $S \leq \underline{S}(\hat{t})$. If $L(\hat{t}) \notin\{\emptyset,[0, \hat{t})\}$, then by iii) and iv) and the mean value theorem there is $\tau \in[0, \hat{t})$ such that $W(\tau \mid S, T)=0$, i.e., another symmetric equilibrium exists under $(S, T)$. The same conclusion is obtained analogously if $U(\hat{t}) \notin\{\emptyset,(\hat{t}, \infty)\}$. Therefore, for $(S, T)$ to implement the symmetric equilibrium with cutoff level $\hat{t}$ when $S \leq \underline{S}(\hat{t})$, it is necessary that $L(\hat{t}) \in\{\emptyset,[0, \hat{t})\}$ and $U(\hat{t}) \in\{\emptyset,(\hat{t}, \infty)\}$. We consider these possibilities below.

If $L(\hat{t})=U(\hat{t})=\emptyset$, then $\underline{S}(\hat{t})=0$. Hence, $S=0$ is the only possible value of $S$ not exceeding $\underline{S}(\hat{t})$. Then, $T=0$ and we showed already that multiple symmetric equilibria exist in this case.

If $L(\hat{t})=[0, \hat{t})$, we must have $W(t \mid S, T)<0$ for all $t<\hat{t}$ for unique implementation of $\hat{t}$ : otherwise, another symmetric equilibrium exists due to iii) and the mean value theorem. Since $W(0 \mid S, T)=S-T$, this would imply $S-T \leq 0$. Then, the null equilibrium would be another symmetric equilibrium under $(S, T)$.

Finally, consider the case $L(\hat{t})=\emptyset$ and $U(\hat{t})=(\hat{t}, \infty)$. For unique implementation of $\hat{t}, W(t \mid S, T)>0$ should hold for all $t>\hat{t}$ : otherwise, another symmetric equilibrium exists due to vii) and the mean value theorem. Then, another equilibrium exists in which every agent adopts regardless of his type. ${ }^{9}$

Proof of Theorem 2: In light of Theorem 1, we only need to show that no asymmetric equilibrium exists under $(S, T)$ if $S$ is sufficiently large. Denote an asymmetric equilibrium, if exists, by $\mathbf{t}=\left(t_{1}, \cdots, t_{N+1}\right) \in \Re_{+}^{N+1}$ where $t_{i}$ is the equilibrium cutoff level of agent $i$. Without loss of generality, assume $t_{1} \leq \cdots \leq t_{N+1}$ and $t_{1}<t_{N+1}$. First we derive conditions that such equilibria must satisfy.

9 The cost of inducing this equilibrium, if possible, is large so that this equilibrium is neither profitmaximizing for an entrepreneur nor a social optimum. 
Let $\mathbf{t}_{-i}:=\left(t_{1}, \cdots, t_{i-1}, t_{i+1}, \cdots, t_{N+1}\right) \in \Re^{N}$. Let $P_{i}(\mathbf{t}) \geq 0$ denote the probability that agent $i$ wins the prize $S$ when he adopts, given that all other agents use the cutoff strategies represented by $\mathbf{t}_{-i}$. Note that this probability does not depend on his own cutoff level $t_{i}$. Specifically, $P_{i}(\mathbf{t}):=\sum_{n=0}^{N} \operatorname{Prob}\left(n \mid \mathbf{t}_{-i}\right) \frac{1}{n+1}$ where $\operatorname{Prob}\left(n \mid \mathbf{t}_{-i}\right)$ is the probability that $n$ other agents adopt given their cutoff strategies $\mathbf{t}_{-i}$. The expected utility of agent $i$ when he is of type $t_{i}$ and adopts, given $\mathbf{t}_{-i}$, is $V^{i}(\mathbf{t}):=\sum_{n=0}^{N} \operatorname{Prob}\left(n \mid \mathbf{t}_{-i}\right) v_{t_{i}}(n)$; the expected net transfer to him is $P_{i}(\mathbf{t}) S-T=\left(P_{i}(\mathbf{t})-P_{A}(\hat{t})\right) S-V(\hat{t})$. For $\mathbf{t}$ to be an equilibrium under $(S, T)$, therefore,

$$
V^{i}(\mathbf{t})-V(\hat{t})+\left(P_{i}(\mathbf{t})-P_{A}(\hat{t})\right) S=0 \quad \forall i=1, \cdots, N+1 .
$$

Next we show that asymmetric equilibrium does not exist under $(S, T)$ if $S$ is sufficiently large. To reach a contradiction, suppose to the contrary that for a sequence $\left\{S_{k}\right\}$ with $\lim _{k \rightarrow \infty} S_{k}=\infty$, asymmetric equilibrium $\mathbf{t}\left(S_{k}\right)=\left(t_{1}\left(S_{k}\right), \cdots, t_{N+1}\left(S_{k}\right)\right)$ exists under $\left(S_{k}, T_{k}\right)$ for each $k$ where $T_{k}=V(\hat{t})+P_{A}(\hat{t}) \cdot S_{k}$. By taking a subsequence if necessary, assume without loss of generality that $\lim _{k \rightarrow \infty} t_{i}\left(S_{k}\right) \in \Re^{+} \cup\{\infty\}$ for all $i=1, \cdots, N+1$.

Since $v_{t}$ is bounded so is $V^{i}(\mathbf{t})$. For (11) to hold for all $S_{k}$, therefore, we need $\lim _{k \rightarrow \infty} P_{i}\left(\mathbf{t}\left(S_{k}\right)\right)=P_{A}(\hat{t})$ for all $i$. This is possible only if $\lim _{k \rightarrow \infty} t_{i}\left(S_{k}\right)=\hat{t}$ for all $i$, i.e., $\lim _{k \rightarrow \infty} \mathbf{t}\left(S_{k}\right)=\hat{\mathbf{t}}:=(\hat{t}, \cdots, \hat{t}) \in \Re^{N+1}$. To see this, note that if $\lim _{k \rightarrow \infty} t_{1}\left(S_{k}\right) \neq \hat{t}$, then

i) $\lim _{k \rightarrow \infty} t_{1}\left(S_{k}\right)<\hat{t}$ for otherwise $\lim _{k \rightarrow \infty} P_{1}\left(\mathbf{t}\left(S_{k}\right)\right)<P_{A}\left(\lim _{k \rightarrow \infty} t_{1}\left(S_{k}\right)\right)<P_{A}(\hat{t})$;

ii) $\lim _{k \rightarrow \infty} t_{N+1}\left(S_{k}\right)>\hat{t}$ for otherwise $\lim _{k \rightarrow \infty} P_{N+1}\left(\mathbf{t}\left(S_{k}\right)\right)>P_{A}(\hat{t})$ would follow because $\lim _{k \rightarrow \infty} t_{1}\left(S_{k}\right)<\hat{t}$ and $\lim _{k \rightarrow \infty} t_{j}\left(S_{k}\right) \leq \hat{t}$ for all $j \neq 1$.

This would mean that $P_{1}\left(\mathbf{t}\left(S_{k}\right)\right)-P_{N+1}\left(\mathbf{t}\left(S_{k}\right)\right)$ is bounded away from 0 , so that it would not be possible for both $P_{1}\left(\mathbf{t}\left(S_{k}\right)\right)$ and $P_{N+1}\left(\mathbf{t}\left(S_{k}\right)\right)$ to converge to $P_{A}(\hat{t})$ as required. Hence, we conclude that $t_{1}\left(S_{k}\right)$ should converge to $\hat{t}$, and analogously, so should $t_{N+1}\left(S_{k}\right)$. Consequently, $\mathbf{t}\left(S_{k}\right)$ should converge to $\hat{\mathbf{t}}$. Now we show that this is impossible.

Since $V^{1}\left(\mathbf{t}\left(S_{k}\right)\right)>V^{N+1}\left(\mathbf{t}\left(S_{k}\right)\right)$, (11) implies

$$
-\frac{V^{i}\left(\mathbf{t}\left(S_{k}\right)\right)-V(\hat{t})}{\Pi^{i}\left(\mathbf{t}\left(S_{k}\right)\right)}=S_{k} \quad \forall k
$$

where $\Pi^{i}(\mathbf{t}):=P_{i}(\mathbf{t})-P_{A}(\hat{t})$. Define $\mathbf{V}: \Re^{N+1} \rightarrow \Re^{N+1}$ and $\Pi: \Re^{N+1} \rightarrow \Re^{N+1}$ as $\mathbf{V}(\mathbf{t}):=\left(V^{1}(\mathbf{t})-V(\hat{t}), \cdots, V^{N+1}(\mathbf{t})-V(\hat{t})\right)$ and $\Pi(\mathbf{t}):=\left(\Pi^{1}(\mathbf{t}), \cdots, \Pi^{N+1}(\mathbf{t})\right)$. Since $v_{t}(n)$ and $F$ are differentiable in $t$ by assumption, so are $\mathbf{V}$ and $\Pi$. Let $D \mathbf{V}(\hat{\mathbf{t}})$ and $D \Pi(\hat{\mathbf{t}})$ denote the derivative of $\mathbf{V}$ and $\Pi$, respectively, evaluated at $\hat{\mathbf{t}}$ : these are $(N+1) \times(N+1)$ Jacobian matrices of $\mathbf{V}$ and $\Pi$, respectively, evaluated at $\hat{\mathbf{t}}$. Note that $\mathbf{V}(\hat{\mathbf{t}})=0$ by definition and $\Pi(\hat{\mathbf{t}})=0$ by construction. Hence, according to the definition of derivative (see, e.g., Spivak (1965) p.16),

$$
\begin{aligned}
& \mathbf{V}\left(\mathbf{t}\left(S_{k}\right)\right)=D \mathbf{V}(\hat{\mathbf{t}}) \cdot\left(\mathbf{t}\left(S_{k}\right)-\hat{\mathbf{t}}\right)+o_{V}\left(\mathbf{t}\left(S_{k}\right)-\hat{\mathbf{t}}\right) \quad \text { and } \\
& \Pi\left(\mathbf{t}\left(S_{k}\right)\right)=D \Pi(\hat{\mathbf{t}}) \cdot\left(\mathbf{t}\left(S_{k}\right)-\hat{\mathbf{t}}\right)+o_{\Pi}\left(\mathbf{t}\left(S_{k}\right)-\hat{\mathbf{t}}\right)
\end{aligned}
$$

where $o_{V}: \Re^{N+1} \rightarrow \Re^{N+1}$ and $o_{\Pi}: \Re^{N+1} \rightarrow \Re^{N+1}$ are two "negligible" functions such that $\frac{\left|o_{V}(\mathbf{t})\right|}{|\mathbf{t}|} \rightarrow 0$ as $|\mathbf{t}| \rightarrow 0$ and similarly for $o_{\Pi}$. (Here, $|\mathbf{t}|$ is the usual norm of $\Re^{N+1}$.) 
Note that $D \Pi(\hat{\mathbf{t}})$ is a symmetric square matrix with 0 as its diagonal elements and $\alpha<0$ as off-diagonal elements where $\alpha$ is the derivative of $P_{i}(\hat{\mathbf{t}})$ with respect to $t_{j}$ for $j \neq i$, i.e., the marginal effect on the probability that agent $i$ wins the prize (if he adopts) when an agent $j \neq i$ increases his cutoff level from $\hat{\mathbf{t}}$. As such, $D \Pi(\hat{\mathbf{t}})$ is clearly nonsingular, i.e., it represents a linear transformation of $\Re^{N+1}$ that is one-to-one and onto. ${ }^{10}$ Hence, $\left|D \Pi(\hat{\mathbf{t}}) \cdot\left(\mathbf{t}\left(S_{k}\right)-\hat{\mathbf{t}}\right)\right| \geq \theta\left|\mathbf{t}\left(S_{k}\right)-\hat{\mathbf{t}}\right|$ where $\theta:=\min _{\{\mathbf{t}|| \mathbf{t} \mid=1\}}|D \Pi(\hat{\mathbf{t}}) \cdot \mathbf{t}| /|\mathbf{t}|$ is strictly positive because $D \Pi(\hat{\mathbf{t}})$ is nonsingular. $D \mathbf{V}(\hat{\mathbf{t}})$ also represents a linear transformation of $\Re^{N+1}$ (not necessarily onto), hence $\left|D \mathbf{V}(\hat{\mathbf{t}}) \cdot\left(\mathbf{t}\left(S_{k}\right)-\hat{\mathbf{t}}\right)\right| \leq \Theta\left|\mathbf{t}\left(S_{k}\right)-\hat{\mathbf{t}}\right|$ where $\Theta:=\max _{\{\mathbf{t}|| \mathbf{t} \mid=1\}} \mid D \mathbf{V}(\hat{\mathbf{t}})$. $\mathbf{t}|/| \mathbf{t} \mid \geq 0$. Therefore,

$$
\begin{aligned}
\lim _{k \rightarrow \infty} \frac{\left|\mathbf{V}\left(\mathbf{t}\left(S_{k}\right)\right)\right|}{\left|\Pi\left(\mathbf{t}\left(S_{k}\right)\right)\right|} & =\lim _{k \rightarrow \infty} \frac{\frac{\left|D \mathbf{V}(\hat{\mathbf{t}}) \cdot\left(\mathbf{t}\left(S_{k}\right)-\hat{\mathbf{t}}\right)+o_{V}\left(\mathbf{t}\left(S_{k}\right)-\hat{\mathbf{t}}\right)\right|}{\left|\mathbf{t}\left(S_{k}\right)-\hat{\mathbf{t}}\right|}}{\frac{\left|D \Pi(\hat{\mathbf{t}}) \cdot\left(\mathbf{t}\left(S_{k}\right)-\hat{\mathbf{t}}\right)+o_{\Pi}\left(\mathbf{t}\left(S_{k}\right)-\hat{\mathbf{t}}\right)\right|}{\left|\mathbf{t}\left(S_{k}\right)-\hat{\mathbf{t}}\right|}} \\
& =\lim _{k \rightarrow \infty} \frac{\frac{\left|D \mathbf{V}(\hat{\mathbf{t}}) \cdot\left(\mathbf{t}\left(S_{k}\right)-\hat{\mathbf{t}}\right)\right|}{\left|\mathbf{t}\left(S_{k}\right)-\hat{\mathbf{t}}\right|}}{\frac{\left|D \Pi(\hat{\mathbf{t}}) \cdot\left(\mathbf{t}\left(S_{k}\right)-\hat{\mathbf{t}}\right)\right|}{\left|\mathbf{t}\left(S_{k}\right)-\hat{\mathbf{t}}\right|}} \leq \frac{\Theta}{\theta}<\infty .
\end{aligned}
$$

This is a contradiction to (12) because (12) implies that $\mathbf{V}\left(\mathbf{t}\left(S_{k}\right)\right)=-S_{k} \Pi\left(\mathbf{t}\left(S_{k}\right)\right)$ and, therefore, $\lim _{k \rightarrow \infty}\left|\mathbf{V}\left(\mathbf{t}\left(S_{k}\right)\right)\right| /\left|\Pi\left(\mathbf{t}\left(S_{k}\right)\right)\right|=\infty$. This contradiction establishes that asymmetric equilibrium does not exist under $(S, T)$ if $S$ is sufficiently large.

\section{References}

Andreoni, J. (1998), "Toward a theory of charitable fund-raising," Journal of Political Economy 106 1186-1213.

Bagnoli, M. and Lipman, B. (1989), "Provision of public goods: Fully implementing the Core through private contributions," Review of Economic Studies 56, 583-601.

Dybvig, P. and Spatt, C. (1983), "Adoption externalities as public goods," Journal of Public Economics 20, 231-247.

Gale, D. (1995), "Dynamic coordination games," Economic Theory 5 1-18.

Gradstein, M. (1992), "Time dynamics and incomplete information in the private provision of public goods," Journal of Political Economy 100 581-597.

Farrell, J. and Saloner, G. (1985), "Standardization, compatibility, and innovation," Rand Journal of Economics 16, 70-83.

Marx, L. and Matthews, S. (2000), "Dynamic voluntary contribution to a public project," Review of Economic Studies 67 327-358.

Rohlfs, J. (1974), "A theory of interdependent demand for a communication service," Bell Journal of Economics 5, 16-37.

Spivak, M. (1965), Calculus on Manifolds, New York, NY: W. A. Benjamin, Inc.

\footnotetext{
10 Note that $P_{i}(\mathbf{t})$ is the probability that agent $i$ wins the prize given that he adopts, so $\sum_{i=1}^{N+1} P_{i}(\mathbf{t}) \neq 1$ generally, hence $\sum_{i=1}^{N+1} \Pi(\mathbf{t}) \neq 0$ generally.
} 
Figure 1

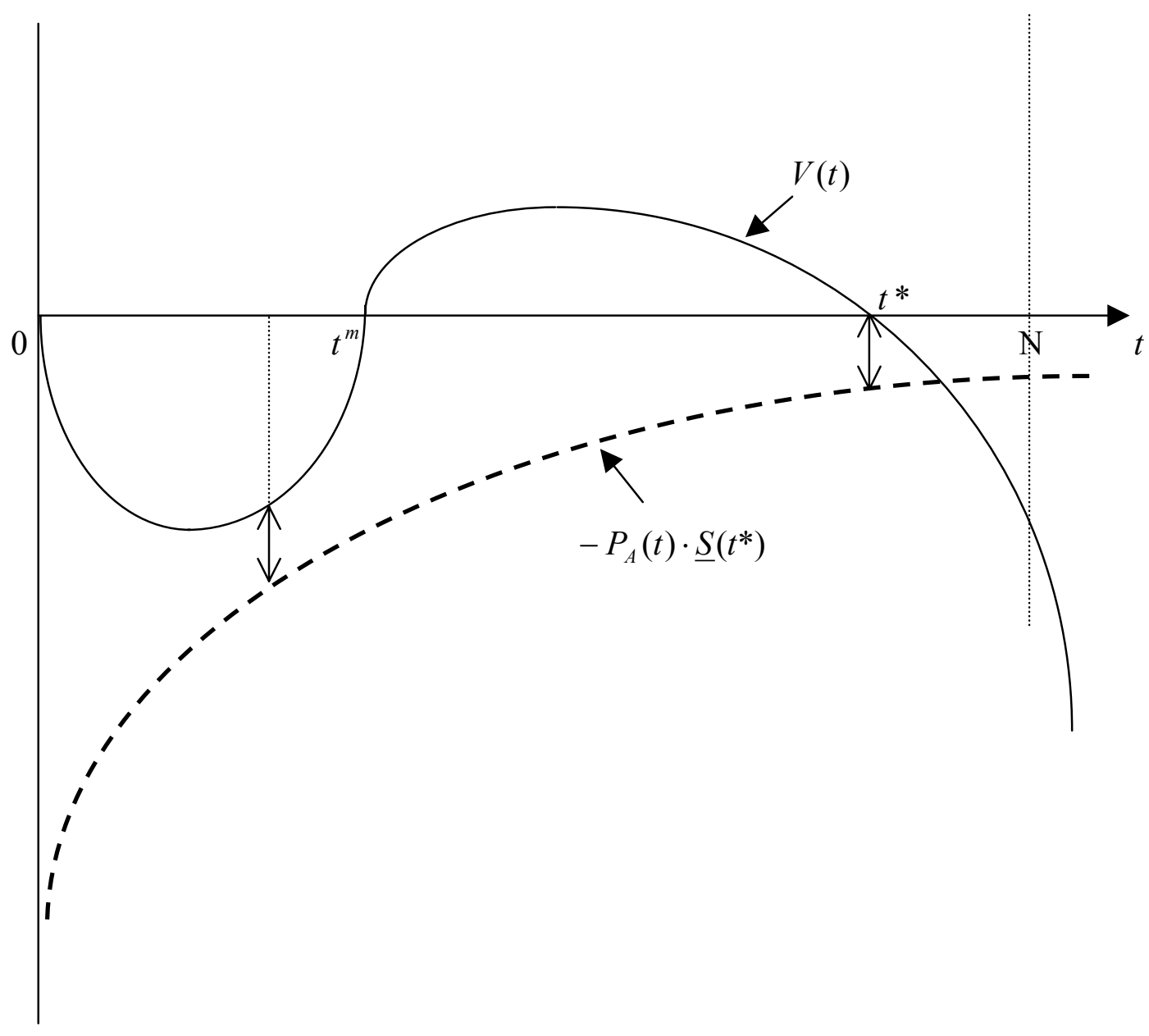

